\title{
Molecular Mobility under Nanometer Scale Confinement
}

\author{
Taek-Soo Kim, and Reinhold H. Dauskardt* \\ Department of Materials Science and Engineering, Stanford University, Stanford, California 94305
}

\begin{abstract}
The mobility of organic molecules under nanoscale confinement differs greatly from that in the bulk. In this study we show that the conventional free volume dependent mobility relationship explained by the free volume theory of diffusion breaks down for diffusion of linear alkane molecules in organosilicate films with connected nanoporosity. Alkane mobility under such nanoscale confinement was observed to decrease with chain length and was lower than that reported in the bulk. While the activation energy for diffusion was similar to that in the bulk, it was found to decrease with chain length exactly opposite to the trend observed in the bulk. This suggests an increasing molecular free volume with chain length. The effects of molecular polarity and pore size on diffusion were also demonstrated. Molecular mobility was found to be suppressed with increasing molecular polarity and decreasing pore size.
\end{abstract}

KEYWORDS Molecular mobility, nanoporous, nanoconfinement

$\mathrm{T}$ he mobility of organic molecules when confined at nanometer length scales differs greatly from properties in the bulk. ${ }^{1,2}$ We demonstrate that linear alkane molecules exhibit a free volume dependent mobility under nanoconfinement that is exactly opposite to the conventional relationship observed in the bulk. As described by the free volume theory of diffusion, ${ }^{3-5}$ linear alkane molecules typically exhibit a lower free volume and mobility with longer chain lengths. ${ }^{6}$ However, under nanoscale confinement in nanoporous organosilicate films, we found the activation energy decreases with increasing chain length suggesting an increase in the molecular free volume.

The effects of such nanoscale confinement on molecular mobility are attracting increasing interest for applications such as pore filling in solid-state dye-sensitized solar cells ${ }^{7}$ and molecular sieving in nanofilters. ${ }^{8}$ However, there is a remarkable paucity of molecular mobility data under nanoconfinement, and fundamental descriptions are complicated not only by the effects of the confinement on molecular interactions, conformation, and free volume but also by molecular interactions with the confining surfaces. We recently showed that unentangled surfactant molecules in the bulk become entangled with adjacent molecules when diffusing through interconnected nanopores $(d \sim 2.1 \mathrm{~nm})$ and exhibit signatures of reptation. ${ }^{9}$ We also discussed the importance of molecular interactions with pore surfaces. Other studies have shown that long polystyrene molecules with molecular weight above that needed for entanglement diffuse faster in cylindrical alumina nanopores

*To whom correspondence should be addressed, dauskardt@stanford.edu. Received for review: 04/03/2010

Published on Web: 04/14/2010 (pore diameter $d \sim 15 \mathrm{~nm}$ ) due to lower molecular entanglement associated with fewer chains present in the confined region. ${ }^{10}$

According to the free volume theory of diffusion, ${ }^{3-5}$ the two ends of a linear chain molecule provide a contribution to free volume into which segments of adjacent molecules can move. The fractional free volume, $f$, provided by the chain ends is inversely proportional to the chain length or the molecular weight, $M$, and increasing $f$ enhances the molecular mobility at low $M$. In the present study, we demonstrate that this free volume dependent classical mobility relationship breaks down for nonpolar linear alkanes under nanoscopic confinement in a strongly hydrophobic nanoporous organosilicate film. We further report on the effect of molecular polarity on the mobility by comparison with mobility of linear fatty alcohols, which are equivalent to the alkanes with a terminal hydrogen atom replaced by a polar hydroxyl group $(-\mathrm{OH})$. The effect of pore diameter on the mobility of the fatty alcohols in nanoporous organosilicate thin films was also demonstrated.

A nanoporous methylsilsesquioxane (MSSQ) film was deposited on a Si substrate by spin-on coating and cured by broad-band UV radiation. The final film thickness, $h \sim 500$ $\mathrm{nm}$, density, $\rho \sim 1.06 \mathrm{~g} / \mathrm{cm}^{3}$, pore diameter, $d \sim 2.1 \mathrm{~nm}$, and dielectric constant, $k \sim 2.3$, were measured. ${ }^{9}$ The MSSQ glass matrix is a structurally modified form of silica produced by replacing one bridging oxygen atom with a terminal methyl group yielding a film with reduced $k$ and strong hydrophobicity (contact angle with water $>100^{\circ}$ ). Organosilicate glass (OSG) and porogen-containing organosilicate glass (pOSG) films with similar composition but more disordered molecular structure were deposited on Si substrates by plasma-enhanced chemical vapor deposition (PECVD) and cured with broad-band UV radiation. The cured OSG and pOSG films were $\sim 500 \mathrm{~nm}$ thick and had pore diameters $d$ 
$\sim 0.8$ and $1.4 \mathrm{~nm}$, respectively. The OSG and MSSQ films were inherently porous, and the pOSG film contained an organic sacrificial porogen (pore generator) that was removed by postdeposition UV curing. The UV curing was designed to promote cross-linking of the $\mathrm{Si}-\mathrm{O}$ network bonds and accelerate porogen removal resulting in the formation of nanometer size pores within the film. ${ }^{11}$

Diffusion coefficients of linear alkanes with various chain lengths from hexane $\left(\mathrm{C}_{6} \mathrm{H}_{14}\right)$ to hexadecane $\left(\mathrm{C}_{16} \mathrm{H}_{34}\right)$, and linear fatty alcohols from hexanol $\left(\mathrm{C}_{6} \mathrm{H}_{13} \mathrm{OH}\right)$ to undecanol $\left(\mathrm{C}_{11} \mathrm{H}_{23} \mathrm{OH}\right)$, were measured using the lateral solvent diffusion technique. ${ }^{9,12}$ To directly observe liquid penetration in the plane of the nanoporous film, the top surface of the film was first hermetically sealed with an optically transparent SiN layer. The silicon substrates containing the films were cleaved to expose a fresh through-thickness surface of the film, and immersed in the liquid of interest. Penetration of the liquid into the plane of the film from the freshly exposed film surface resulted in a change in the refractive index and color of the film that could be easily monitored by optical observation through the top surface of the films using an optical microscope. Controlled temperatures of 22, 31, and $42 \pm 1{ }^{\circ} \mathrm{C}$ were used.

We note that there are two fundamental approaches for modeling fluid penetration in porous media, namely, the Washburn capillary flow model ${ }^{13}$

$$
x=\sqrt{\frac{d \gamma \cos \theta}{4 \eta} t}
$$

where $x$ is the penetration distance, $d$ is the channel diameter, $\gamma$ is the surface tension, $\theta$ is the contact angle, $\eta$ is the viscosity, and $t$ is the penetration time. The other is a diffusion model ${ }^{9,12}$

$$
x=\sqrt{D t}
$$

where $D$ is the Fickian collective diffusion coefficient. Both models exhibit the same square root of time dependence and have been shown to yield equivalent results for fluid penetration in porous media. ${ }^{14-16}$ Therefore the prefactor of $t$ in eq 1 may be regarded as $D$ by analogy. A significant consideration for the Washburn model is that the viscosity, $\eta$, cannot be assumed to be constant under nanometer scale confinement due to increasing liquid-surface interactions. ${ }^{1,2}$ Of greater concern, however, is if the Washburn model is applicable at the nanoscale. When the confining channel size approaches the dimension of the fluid molecules themselves, it is questionable whether the capillary flow model which includes a net force related to a fluid meniscus in contact with channel walls is physically realistic. Assigning an appropriate contact angle, $\theta$, may not be meaningful given the complexities of fluid/solid contact at nanometer length scales.

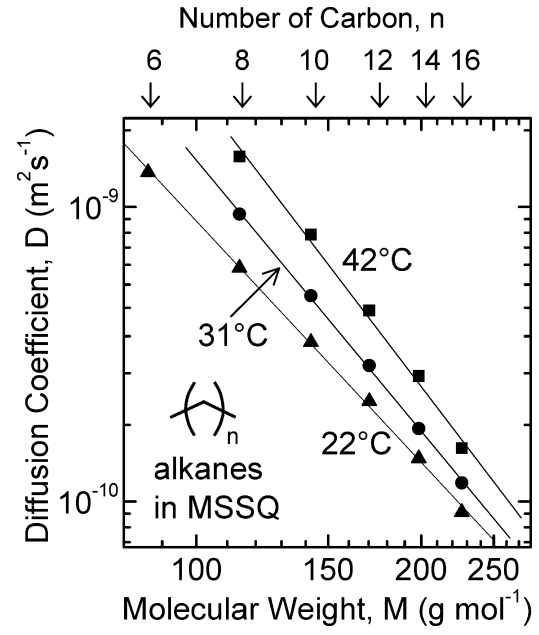

FIGURE 1. Diffusion coefficients of the alkanes in the MSSQ film were measured at temperatures of 22,31 , and $42{ }^{\circ} \mathrm{C}$ and plotted as a function of molecular weight on a $\log -\log$ scale.

The Fickian-like diffusion does not rely on any assumptions about a meniscus surface contact driving force. The diffusion coefficient, $D$, which is unique for each liquid-pore system, contains all the information regarding the changes of molecular mobility under nanometer scale confinement. Of course, the molecular mobility as described through $D$ is related to $\eta$ at a fundamental level and, as we discuss later, both are significantly affected by nanoscale confinement. To some degree, however, it does not matter whether we envisage a diffusion or capillary flow model to interpret the principal results of the study as discussed in the following sections.

Diffusion coefficients of alkanes in the nanoporous MSSQ film $(d \sim 2.1 \mathrm{~nm})$ were measured at selected temperatures and plotted as a function of alkane molecular weight (Figure 1). The diffusion coefficient, $D$, decreased significantly with decreasing temperature and increasing molecular weight. $D$ scaled with the molecular weight $M$ according to $D \sim M^{-\alpha} .{ }^{17}$ The diffusivities in the MSSQ film were lower than those reported in the bulk by at least a factor of $3 .{ }^{6}$ Moreover, the exponent $\alpha$ increased with temperature with values of 2.72 at $22{ }^{\circ} \mathrm{C}, 3.12$ at $31^{\circ} \mathrm{C}$, and 3.31 at $42{ }^{\circ} \mathrm{C}$ as evidenced by the increasing slope in Figure 1. This is opposite to the trend for these alkanes in the bulk where the slope $\alpha$ decreased with temperature. ${ }^{6}$

The diffusion coefficients were plotted in terms of the inverse temperature in Figure 2a, from which the activation energies for diffusion in the MSSQ film were calculated using the Arrhenius equation, $D=D_{0} \exp \left(-E_{\mathrm{a}} / k_{\mathrm{B}} T\right)$, where $D_{0}$ is a constant, $E_{\mathrm{a}}$ is the activation energy, $k_{\mathrm{B}}$ is Boltzmann's constant, and $T$ the absolute temperature. Figure $2 \mathrm{~b}$ shows the calculated activation energies as a function of the number of the carbon atoms of the alkane molecule together with reported activation energies in the bulk. ${ }^{6}$ The activation energy was found to decrease with chain length under nanoscale confinement, exactly opposite to that observed in the bulk. ${ }^{6}$ 

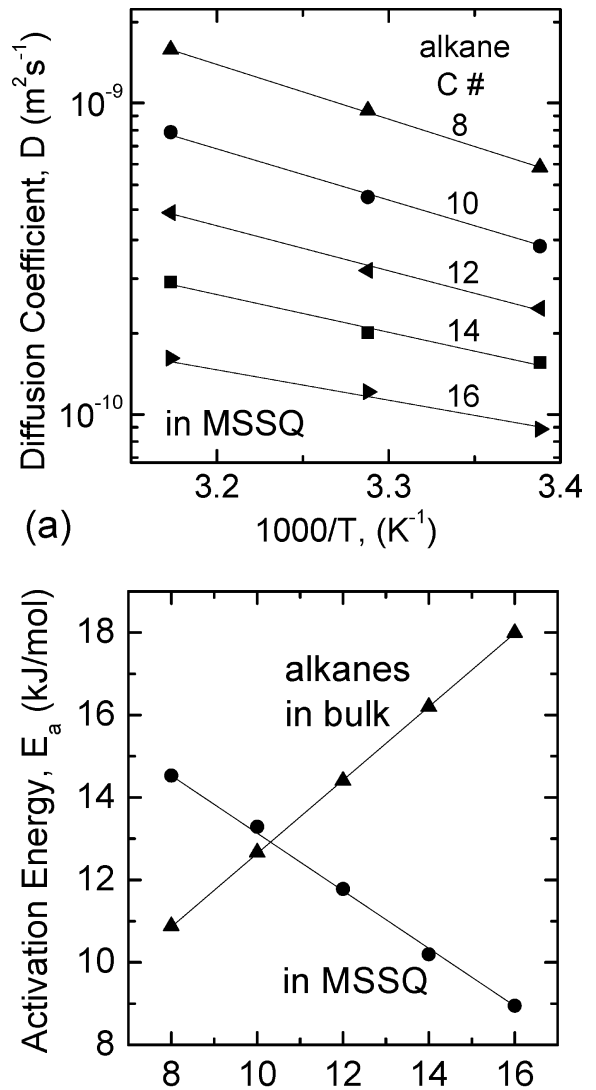

(b) Number of Carbon, $n$

FIGURE 2. The diffusion of alkanes in the MSSQ film showing (a) the diffusion coefficients as a function of the inverse temperature on a log-linear scale and (b) the resulting activation energies plotted as a function of the carbon number of the alkane molecule and compared with the activation energies measured in the bulk. ${ }^{6}$

The reason for the increasing activation energy with chain length in the bulk is related to the molecular chain end effects on free volume. ${ }^{3-6}$ Melts of longer alkane chains have fewer chain ends per unit volume meaning less local free volume into which the segments of adjacent molecules can move. The resulting reduction in the free volume leads to the increased activation energy and the decreased mobility. 5,6 Using positron annihilation lifetime spectroscopy and neutron scattering spectroscopy, it has recently been reported that polar glycerol has a higher average free volume and a lower mobility when confined in hydrophilic Vycor pores ( $d$ $\sim 7 \mathrm{~nm}){ }^{18}$ A hydrogen-bonded interfacial layer of glycerol on the pore surfaces is thought to be transmitted through the hydrogen-bonded molecular network to inner regions where relaxation is inhibited and free volume is correspondingly higher. The confined liquid appears to be perturbed to the extent that the motion of molecules is reduced and the classical free volume dependent mobility relationship is reversed. ${ }^{18}$

Our study demonstrates that even nonpolar alkanes may have this reversed relationship in strongly hydrophobic nanopores. Although we were not able to directly measure the molecular free volume, the decreasing activation energy

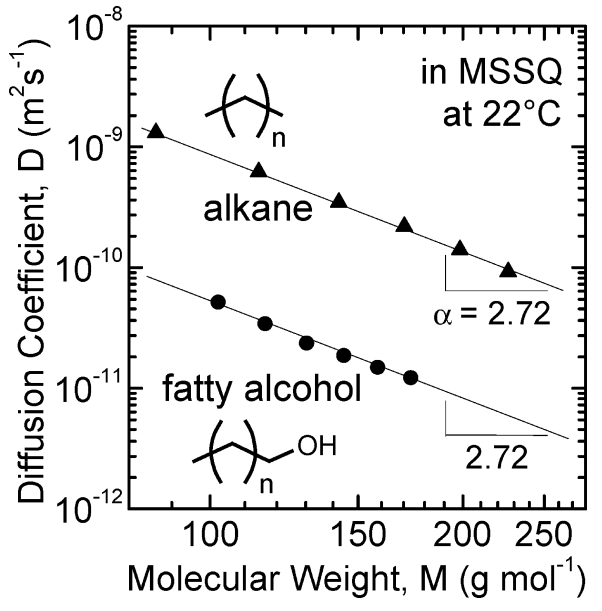

FIGURE 3. Diffusion coefficients of alkanes and fatty alcohols in the MSSQ film at $22^{\circ} \mathrm{C}$ as a function of molecular weight on a log-log scale.

observed with longer alkane chain length (Figure 2b) strongly suggests a similarly perturbed molecular free volume under nanoconfinement. Methyl terminal groups $\left(-\mathrm{CH}_{3}\right)$ on the pore surfaces in the MSSQ film have a much weaker van der Waals attraction with the alkane molecules compared to the polar glycerol interactions. However, relaxation of the alkane molecular conformation in the much smaller MSSQ pores $(d \sim 2.1 \mathrm{~nm})$ was nevertheless inhibited and free volume increased. Since the van der Waals interaction is proportional to the chain length, we would expect more constraint on the molecular relaxation with increasing chain length. This decreases the molecular mobility with chain length (Figure 1) but at the same time increases the free volume so that the activation energy for diffusion decreases under such nanoscale confinement (Figure 2b). These trends are opposite to the classical mobility dependent free volume relationship in the bulk.

We next wanted to determine the effects of increasing the polar character of molecules on molecular mobility in the MSSQ films. We selected fatty alcohols, which are equivalent to the linear alkanes with a terminal hydrogen atom replaced by a hydroxyl group $(-\mathrm{OH})$. While showing exactly the same slope $\alpha=2.72$, the measured diffusion coefficients were found to be significantly lower than those of the alkanes by a factor of $\sim 17$ at the same $M$ (Figure 3). This was attributed to the higher viscosity of fatty alcohols compared to alkanes that result from hydrogen bonding of the polar hydroxyl group. For example, heptanol has higher viscosity than octane by a factor of 13 although they have similar molecular weight, $M \sim 115 \mathrm{~g} / \mathrm{mol}$. The observed relationship between diffusion coefficient and viscosity is consistent with the Stokes-Einstein relation, $D \sim k_{\mathrm{B}} T / \eta$, where the diffusion coefficient is inversely proportional to viscosity, $\eta$.

The effect of the pore diameter on the mobility of organic molecules was also studied. The diffusion coefficients of the fatty alcohols with various chain lengths in nanoporous 


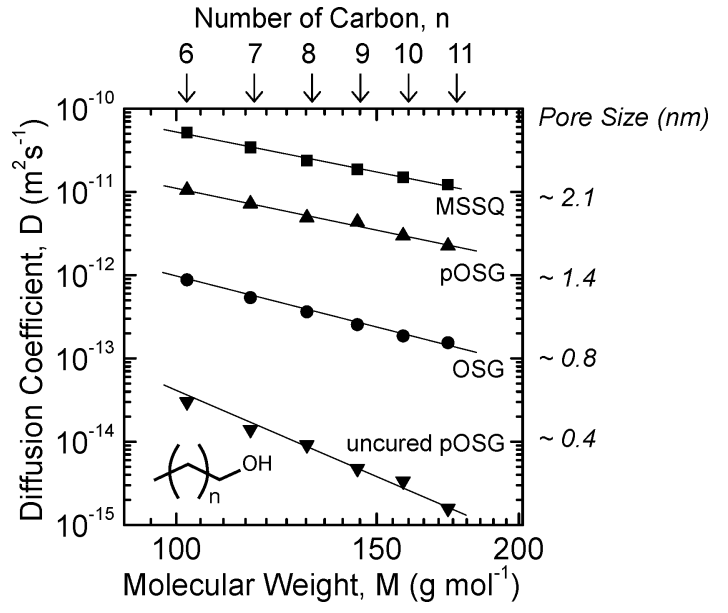

FIGURE 4 . The diffusion coefficients of fatty alcohols with various chain lengths in nanoporous organosilicate films with selected pore sizes were plotted as a function of molecular weight on a $\log -\log$ scale.

organosilicate films with selected pore sizes are plotted as a function of the molecular weight in Figure 4. A dramatic decrease in diffusion coefficient with decreasing pore diameter was observed together with an increasing sensitivity to molecular weight for smaller pores. To better appreciate the nanoscale confinement effects, the diffusion coefficients were normalized by the pore diameter, $d$, and replotted in Figure $5 \mathrm{a}$ as a function of $d$. Note that if we equate the prefactors of eqs 1 and 2 , then $D \sim d$ and the Washburn capillary flow relationship would appear as a horizontal line as indicated by the dotted line in the figure assuming that the viscosity $\eta$ is not a function of nanoscale confinement. However, clearly $D$ is a strong function of $d$ and this suggests that $\eta$ under such nanoscale confinement should increase significantly with decreasing $d$. Under these conditions we expect $\eta \sim d / D$ by again equating the prefactors of eqs 1 and 2. In Figure $5 \mathrm{~b}$ we show the resulting trend in $\eta$ normalized by $\eta_{2.1}$, where $\eta_{2.1}$ is the viscosity in the largest pore diameter examined $(d \sim 2.1 \mathrm{~nm})$. The striking effect of nanoscale confinement on the apparent viscosity is clear, causing an increase in nearly 3 orders of magnitude as the pore diameter decreased. This dependence of viscosity on confinement length scale below a few nanometers is consistent with other experimental results involving liquids compressed between parallel plates. ${ }^{1,2}$

Another important finding from Figure 4 was that the exponent $\alpha$ continuously increased with the decreasing pore diameter, 2.72 for the $2.1 \mathrm{~nm}$ pore, 2.86 for the $1.4 \mathrm{~nm}$ pore, 3.35 for the $0.8 \mathrm{~nm}$ pore, and 5.35 for the $0.4 \mathrm{~nm}$ pore. This means the diffusivity has a stronger dependence on chain length as the pore size decreases. We attributed this to increasing interactions with the pore surfaces as the pore size decreases and the surface-to-volume ratio of the pores increases. Under these conditions the molecular mobility becomes increasingly affected by van der Waals interactions between the molecules and the methyl groups on pore
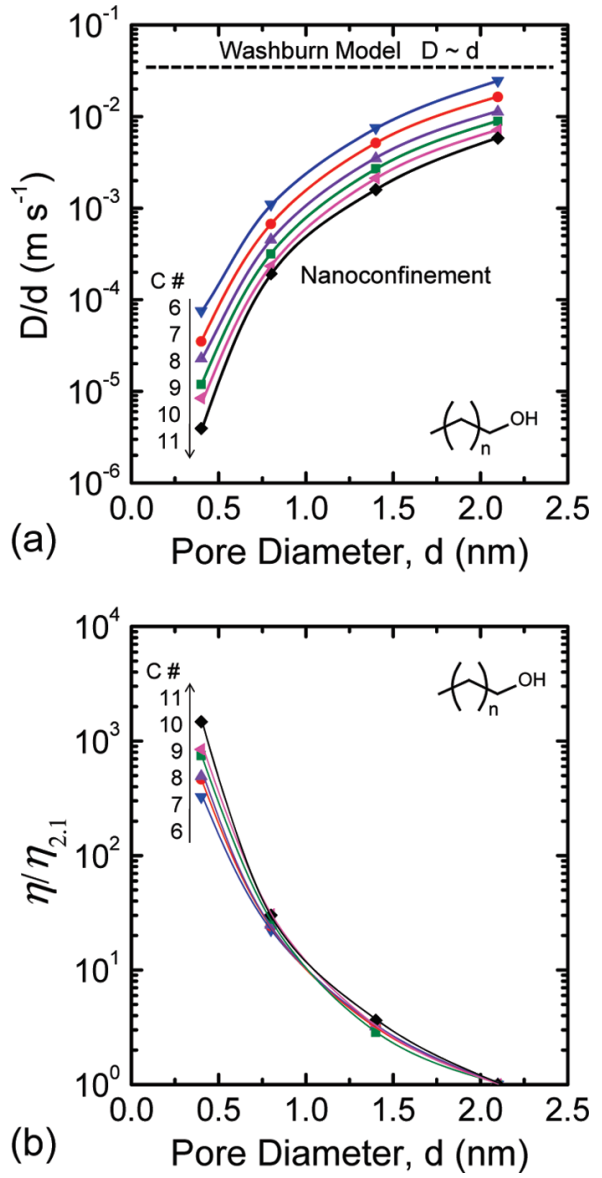

FIGURE 5. (a) The diffusion coefficients, $D$, from Figure 4 were normalized by pore diameter, $d$, and replotted as a function of $d$. The Washburn model is also shown in a dotted line for comparison. (b) The viscosity under nanoconfinement, $\eta$, normalized by the viscosity in the $2.1 \mathrm{~nm}$ pore diameter, $\eta_{2.1}$, plotted as a function of $d$.

surfaces, resulting in the increased chain length dependence of the molecular mobility. The smallest effective pore size was in the uncured POSG film where the sacrificial porogen was not removed. Even under these conditions, however, the fatty alcohols were found to diffuse into the film, although with a molecular mobility that was suppressed by at least 2 orders of magnitude lower compared to the porous pOSG film (Figure 4).

The results have implications for a number of technologies where nanoporous materials are in contact with organic molecules. For example, undesirable diffusion of process environmental species can be minimized by removing the porogen after harsh integration processes. ${ }^{19}$ Fundamental understanding of molecular diffusion under nanometer scale confinement is critical for effective and reliable pore filling in nanoporous $\mathrm{TiO}_{2}$ with conducting polymers for dyesensitized solar cells. ${ }^{7}$ It is also important for mechanical stresses and fracture in nanoporous materials that are shown to be significantly affected by diffusion of environmental species. ${ }^{20,21}$ 
We have measured the mobility of organic alkane molecules in organosilicate films with connected nanoporosity and demonstrated that the conventional mobility dependent free volume relationship explained by the free volume theory of diffusion breaks down under such nanoscale confinement. We further explored the effects of increasing molecular interactions using polar fatty alcohols of similar molecular weight and the effects of molecular pore surface interactions by varying the pore size. The results help elucidate the important roles of molecular type and polarity, molecule/molecule and molecule/surface interactions, and pore size on diffusion under nanoscale confinement.

Acknowledgment. The authors are grateful for support with diffusion tests from LeMoyne Habimana-Griffin and Molong Li. This work was supported by the Director, Office of Energy Research, Office of Basic Energy Sciences, Materials Sciences Division of the U.S. Department of Energy, under Contract No. DE-FG02-07ER46391.

\section{REFERENCES AND NOTES}

(1) Klein, J.; Kumacheva, E. Science 1995, 269, 816-819.

(2) Granick, S. Science 1991, 253, 1374-1379.

(3) Cohen, M. H.; Turnbull, D. J. Chem. Phys. 1959, 31 (5), $1164-$ 1169.

(4) Williams, M. L.; Landel, R. F.; Ferry, J. D.J. Am. Chem. Soc. 1955 77, 3701 .

(5) Macedo, P. B.; Litovitz, T. A. J. Chem. Phys. 1965, 42 (1), 245256.
(6) von Meerwall, E.; Beckman, S.; Jang, J.; Mattice, W. L. J. Chem. Phys. 1998, 108 (10), 4299-4304.

(7) Ding, I.; Tétreault, N.; Brillet, J.; Hardin, B. E.; Smith, E. H.; Rosenthal, S. J.; Sauvage, F.; Grätzel, M.; McGehee, M. D. Adv. Funct. Mater. 2009, 19, 2431-2436.

(8) Han, J.; Fu, J.; Schoch, R. B. Lab Chip 2008, 8 (1), 23-33.

(9) Kim, T.; Mackie, K.; Zhong, Q.; Peterson, M.; Konno, T.; Dauskardt, R. H. Nano Lett. 2009, 9 (6), 2427-2432.

(10) Shin, K.; Obukhov, S.; Chen, J.; Hun, J.; Hwang, Y.; Mok, S.; Dobriyal, P.; Thiyagarajan, P.; Russell, T. Nat. Mater. 2007, 6, 961-965

(11) Gage, D. M.; Stebbins, J. F.; Peng, L.; Cui, Z.; Al-Bayati, A.; MacWilliams, K. P.; M'Saad, H.; Dauskardt, R. H. J. Appl. Phys. 2008, 104, No. 043513.

(12) Guyer, E. P.; Gantz, J.; Dauskardt, R. H. J. Mater. Res. 2007, 22 (3), 710-718

(13) Washburn, E. W. Phys. Rev. 1921, 17, 273-283.

(14) Oliver, J. F.; Agbezuge, L.; Woodcock, K. Colloids Surf., A 1994, $89,213-226$

(15) Ruoff, L.; Prince, D. L.; Giddings, J. C.; Stewart, G. H. Colloid Polym. Sci. 1959, 166 (2), 144.

(16) Ruoff, L.; Stewart, G. H.; Shin, H. K.; Giddings, J. C. Colloid Polym. Sci. 1960, 173 (1), 14.

(17) de Gennes, P. G. Scaling Concepts in Polymer Physics; Cornell University Press: Ithaca and London, 1979.

(18) Kilburn, D.; Sokol, P. E.; Sakai, V. G.; Alam, M. A. Appl. Phys. Lett. 2008, 92, No. 033109.

(19) Jousseaume, V.; Favennec, L.; Zenasni, A.; Passemard, G. Appl. Phys. Lett. 2006, 88, 182908.

(20) Kim, T.; Konno, T.; Dauskardt, R. H. Acta Mater. 2009, 57, 46874696.

(21) Guyer, E. P.; Patz, M.; Dauskardt, R. H. J. Mater. Res. 2006, 21 (4), 882-894. 